\title{
Reflexiones sobre la relación entre las neurociencias y el psicoanálisis"
}

\section{Reflections on the Relationship between Neuroscience and Psychoanalysis}

Recibido: marzo 20 de 2010 | Revisado: abril 26 de 2010 | Aceptado: mayo 6 de 2010

\author{
Sergio CASTELlanos ** \\ Pontificia Universidad Javeriana, Bogotá, Colombia
}

Para citar este artículo. Castellanos, S. (2010). Reflexiones sobre la relación entre las neurociencias y el psicoanálisis. Universitas Psychologica, 9 (3), 729-735.

* Artículo de reflexión teórica.

** Facultad de Psicología, Pontificia Universidad Jave-

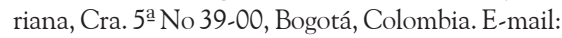
scastellanos@javeriana.edu.co

\section{RES UMEN}

El psicoanálisis se consolidó como ciencia en un momento en el cual los avances de las neurociencias no permitían dar explicación suficiente a ciertos fenómenos psíquicos. Por ello, aun cuando el camino que recorrió Freud partió de la medicina y de las investigaciones neurofisiológicas, éstas no fueron suficientes para comprender fenómenos tan complejos y vigentes, en ese entonces, como el de la histeria. Se presentan algunos elementos significativos del paso de Freud por la medicina y la neurología, en cuanto a limitación en su momento, que lo llevó a prescindir de ellas a través de la metodología investigativa del psicoanálisis, que valdría la pena seguir usando para continuar los desarrollos internos del mismo. Se retoma la resistencia como elemento interferente para no conocer en profundidad el psicoanálisis y atender más bien a los afanes postmodernos de producir conocimiento novedoso -y en muchos casos superficial-, proponer nuevas disciplinas, y no adentrarse en las profundidades de las ya existentes, que aún tienen muchas posibilidades de desarrollos internos.

\section{Palabras clave autor}

Psicoanálisis, neurociencias, neuropsicoanálisis, Freud.

Palabras clave descriptor

Freud, Sigmund, 1856-1939, crítica e interpretación, neurociencias,

neuropsicoanálisis.

\section{A B S T R A C T}

Psychoanalysis was consolidated as a science at a time where the advantages of neuroscience didn't allowed giving satisfactory explications to certain psychological phenomena. This explains that even though roads travel by Freud began at medicine and neurophysiologic investigations, these disciplines were not effective enough to understand phenomena as hysteria. Some of the significant elements taken by Freud from medicine and neurology are presented. The concept of resistance as an element of interference to gain deeper psychoanalytic knowledge is also discussed, and its relation to our preference for the post modern rush of producing novel knowledge, even superficial in some cases, or to propose new disciplines, in order to avoid depth in research and knowledge into already existing disciplines that still have a lot of internal development possibilities.

Keywords author

Psychoanalysis, Neuroscience, Neuropsychoanalysis, Freud.

Keywords plus

Freud, Sigmund, 1856-1939, Criticism and Interpretation, Neurosciences, Neuropsychoanalysis. 
Los avances de cualquier disciplina científica traen evidentes beneficios, para la ciencia en general, pero éstos son más obvios al interior de aquella en la que se han logrado, así como también, resulta más fácil su comprensión para los expertos en tal doctrina. También se puede evaluar si los beneficios de tales avances sobrepasan el interior de tal disciplina, y en qué medida favorecen entonces otras, y en el mejor de los casos, el bienestar general de la humanidad.

Dentro de las llamadas neurociencias, es indudable que a lo largo del siglo XX y en lo que va corrido del XXI, se han logrado avances significativos especialmente en términos de observaciones a través de imágenes que permiten hacer localizaciones cada vez más precisas, así como también, se ha logrado una mayor comprensión subjetiva de ciertas alteraciones. Como lo dice Oliver Sacks, en el prólogo del libro El cerebro y el mundo interior de Solms y Turnbull (2004), "la neurología misma tuvo que evolucionar de una ciencia mecánica que pensaba en términos de funciones y centros determinados [...] a un análisis más dinámico de las dificultades neurológicas en términos de sistemas funcionales" (p.vii).

Otro asunto es la consideración por la manera en que esta evolución neurocientífica ha sobrepasado sus fronteras y, de manera particular, ha beneficiado al psicoanálisis. Es en este sentido en el que se quisiera llamar la atención, pues es bien conocido que el camino seguido por el profesor Sigmund Freud, para descubrir y construir el psicoanálisis, efectivamente pasó por la medicina y por la neurología, y por ende está teñido de elementos de estas disciplinas, pero otro asunto sería que estuviese determinado por éstas.

He podido estudiar, con cierto detenimiento, algunos trabajos que buscan no solo sustentar la pertinencia de una nueva orientación, llamada neuropsicoanálisis, sino que incluso presentan algunos materiales clínicos de ésta. Dentro del material revisado sobresalen: los Estudios clínicos en neuropsicoanálisis de Solms (2005), la propuesta del NeuroAnalysis de Peled (2008), y el artículo Biology and the future psychoanalysis de Kandel (1999). Si bien encuentro interesantes sus propuestas, ellas no siguen con rigor los planteamientos metodológicos del psicoanálisis enunciado por Freud. Pero considero que, antes de hacer una crítica a esos caminos, es pertinente exponer más bien aquello que permite dichas confusiones.

Una problemática como la planteada tiene diferentes alternativas para buscar resolverla. Una de ellas, muy interesante, es a través de los aspectos de la lógica de las ciencias, su arquitectónica y sus raíces epistemológicas. Este camino llevaría a las conocidas discusiones de lo que es ciencia o científico, ciencias duras y blandas, o cómo quizás se ha logrado hacer la mejor transacción a la discusión entre las ciencias exactas y las sociales. Por esta vía, sería necesario sustentar los argumentos que respaldan al psicoanálisis, no solo como ciencia, sino como lo afirmó Freud en múltiples ocasiones, como ciencia natural. En la presentación autobiográfica dice Freud (1925/1979):

Siempre sentí como grave injusticia que no se quisiese dispensar al psicoanálisis el mismo trato que a cualquier otra ciencia natural. Ese rehusamiento se expresó en las más pertinaces objeciones. Al psicoanálisis se le reprocha cada una de sus imperfecciones y lagunas, cuando en verdad una ciencia basada en la observación no puede hacer otra cosa que elaborar una por una sus conclusiones y resolver paso a paso sus problemas. (p. 46)

Otra problemática, igualmente compleja, derivada del posible camino anteriormente planteado, es la referida a la concepción del ser humano desde una perspectiva monista o dualista. Esto nos lleva a la también permanente discusión por la división alma-cuerpo o en lenguaje más cercano a las neurociencias, mente-cerebro. La concepción teórica del hombre del psicoanálisis descubierto y planteado por Freud (que por motivos prácticos es al único al que se hace referencia en este texto) es fundamentalmente monista, en términos de la "sustancia" que nos constituye, en cuanto a que es el principio del placer el que intenta gobernarnos, pero dualista en términos de la existencia del conflicto, y de las diferentes fuerzas que, por efecto del interjuego con la realidad en un contexto y en 
relación con unos objetos, generan contradicción, oposición, contrarios y, en general, polaridades. Esta es la postura de Etcheverry (1979) y colaboradores, equipo traductor al castellano de la edición comercializada por Amorrortu Editores.

Si bien las dos alternativas enunciadas son muy interesantes, requieren de un rigor y una revisión documental que trasciende los alcances del presente artículo y son más propios de una tesis doctoral; por ello, se me hace necesario tomar otra alternativa para poder brindar elementos de reflexión, más que una argumentación sólida que no deje mayores dudas. Se opta entonces por revisar otros aspectos más sencillos.

Haber mencionado el asunto dualista del conflicto y del interjuego que allíse da, orienta el paso a la revisión del concepto de "serie complementaria" que Freud utiliza en múltiples ocasiones a lo largo de su obra. Según Valls (2008), este concepto es una explicación dinámica respecto de las causales de los síntomas neuróticos, pero también sobre la formación y cambios en el funcionamiento general del aparato psíquico, en diferentes momentos de la vida. La primera serie está constituida por lo heredado y las vivencias de la prehistoria infantil. Y a la suma de estos dos elementos los denomina constitucional o predisposición. En relación con esta serie es pertinente traer la cita aclaratoria que el mismo Freud (1912) escribe:

Debemos defendernos en este lugar del reproche, fruto de un malentendido, de que soslayamos la significación de los factores innatos (constitucionales) por haber puesto de relieve las impresiones infantiles. Semejante reproche brota de la estrechez de la necesidad causal de los seres humanos, que, en oposición al modo en que de ordinario está plasmada la realidad, quiere darse por contenta con un único factor causal. El psicoanálisis ha dicho mucho sobre los factores accidentales de la etiología, y poco sobre los constitucionales, pero ello sólo porque acerca de los primeros podía aportar algo nuevo, mientras que respecto de los segundos en principio no sabía más que lo que corrientemente se sabe. Nos negamos a estatuir una oposición de principio entre las dos series de factores etiológicos; más bien, suponemos una regular acción conjugada de ambas para producir el efecto observado. [disposición y azar] determinan el destino de un ser humano; rara vez, quizá nunca, lo hace uno solo de esos poderes. La distribución de la eficiencia etiológica entre ellos sólo se podrá obtener individualmente y en cada caso. La serie dentro de la cual se ordenen las magnitudes cambiantes de ambos factores tendrá también, sin duda, sus casos extremos. Según sea el estado de nuestros conocimientos, apreciaremos de manera diversa la parte de la constitución o del vivenciar en el caso singular, y nos reservamos el derecho de modificar nuestro juicio cuando nuestras intelecciones cambien. (p. 97)

Si bien Freud plantea todos los factores constitucionales como un solo elemento de las series complementarias, allí se incluyen todos los elementos que hacen parte de la constitución del sujeto, bien sean genéticamente heredados o fruto de vicisitudes del desarrollo ontogenético. Frente a éstos, el psicoanálisis no tiene nada que decir (pero por supuesto escucha con atención los avances que allí se dan).

Otra serie que opera de manera semejante a lo constitucional es la referida a las vivencias de la prehistoria infantil. Sobre las impresiones infantiles, o vivencias tempranas del infante, el psicoanálisis ha iluminado su comprensión a través de los conceptos de fijación y represión, y es en esta serie en la cual más aportes ha realizado y está en condiciones de profundizar aún más.

Una tercera serie, son las vivencias actuales, en especial las originadas a partir de la frustración; de que algo no sea o resulte como se estaba deseando. Sobre éstas, tanto la psicología, en general, como el psicoanálisis, confluyen en el aporte de elementos esclarecedores ampliamente divulgados. Entonces, al surgir una vicisitud, una necesidad que en tanto no se satisfaga produce sensaciones de displacer, la respuesta a esto estará mediada por la simultánea presencia de las tres series mencionadas. De la misma manera, cualquier intervención que se haga sobre esta situación, tendrá resultados dependiendo del énfasis que ésta tenga, de la serie en la que centren sus estrategias. Por ejemplo, la 
intervención o la cura, a partir de la aplicación del método psicoanalítico, tendrá mayor dificultad, mayores resistencias, en tanto mayor sea el peso de las vivencias infantiles que hayan sido objeto de la represión o desalojo de la consciencia. Pero si el peso mayor está en los factores innatos, su campo de acción será más reducido. Cuando el peso más significativo está en lo actual, usualmente, el camino hacia la cura es menos complicado.

El concepto de "serie complementaria" permite entonces una buena aproximación a la comprensión del asunto de la relación de beneficio entre los hallazgos de las neurociencias sobre el psicoanálisis, pues cualquier avance sobre una de estas series, beneficia la comprensión y quizás la intervención sobre cualquier alteración; es decir, que entre más permitan las neurociencias comprender los factores constitucionales y mejores posibilidades de intervención sobre ellos tenga, mejor para la humanidad. Sin embargo, la intervención psicoanalítica pone su énfasis en otros elementos. Es decir, el campo de lo neurofisiológico, no es realmente el campo del psicoanálisis ni su objeto de estudio.

¿Por qué tenemos la tendencia a atenuar los límites entre las diferentes disciplinas? Pues, en parte, porque efectivamente estas divisiones son artificiales, son construcciones auxiliares o pedagógicas, para poder abordar la complejidad, en este caso, del funcionamiento psíquico de los humanos. También, por las dificultades para comprender una explicación, cuando usualmente estamos ubicados desde otra perspectiva que conocemos más profundamente. Queremos ampliar su poder explicativo a campos que no han sido originalmente los suyos. Y si continuase por este camino, habría que exponer muchos más aspectos científicos y humanos; pero, en este punto, deseo ampliar a los elementos de reflexión sobre la relación en cuestión, a partir de los análisis del propio Freud acerca de las resistencias frente al psicoanálisis, expuestas por él, en particular, en dos artículos. El primero, de 1917, Una dificultad del psicoanálisis, el segundo, Las resistencias contra el psicoanálisis, de 1925, en los que plantea de manera semejante que una dificultad para que un lector -receptor-comprenda el psicoanálisis, podría estar derivada de una de- ficiencia intelectual, la cual se presentaría frente a cualquier disciplina científica. Sin embargo, Freud precisa que se va a ocupar de otro tipo de problema. Se trata de las dificultades afectivas que impiden sentir empatía, interés o curiosidad frente a ciertos conocimientos que, en tanto incluyen elementos significativos relacionados con nuestros propios conflictos, con nuestras neurosis, activan las contracargas de lo reprimido, de lo desalojado de la conciencia; es decir, activan las resistencias, las cuales se expresan en los conflictos afectivos, ocultos en rechazo cultural, en consideraciones morales, en pretextos de desactualización o antigüedad. Estas resistencias, que aborda Freud en los artículos mencionados, son las primeras que un psicoanalista debe considerar acerca de sus propias dificultades; pero es muy difícil que un lego, que no conoce profundamente como operan, pueda examinarlas, precisamente por su cualidad de ser parte del mecanismo inconsciente de la represión.

Luego de dejar planteado que los problemas más significativos para abordar cuestiones psicoanalíticas pueden venir precisamente de nosotros mismos, de nuestro inconsciente, quiero considerar un último elemento como aporte a la reflexión propuesta. Éste es mucho más evidente, pues se trata de ilustrar que los años de interés y dedicación de Freud a las neurociencias de su época, se prestan para acercar estas corrientes al psicoanálisis, no solo como un paso en el camino para llegar a lo propiamente psicoanalítico, sino que algunos concluyen que, efectivamente, las mantuvo estrechamente relacionadas.

Se ilustrarán algunos aspectos fundamentales del paso de Freud por la medicina y la neurología, para poder comprender que efectivamente fueron significativos y que influyeron en el descubrimiento del psicoanálisis, pero otro asunto sería afirmar que su relación no es solo histórica, sino que es determinante constitucionalmente.

Si bien Freud inicia sus estudios de medicina en 1873 , y en 1893 , como lo considera Etcheverry (1979), se editan las primeras publicaciones psicoanalíticas; esos 20 años entre la medicina, la investigación fisiológica, la neurología y la etiología de la histeria, son un paso o camino para los siguientes 
años -más de 40- dedicados exclusivamente al psicoanálisis. En términos evolutivos, conviene analizar separadamente diferentes momentos de este camino hacia el psicoanálisis. En relación con su escogencia por la medicina, en Presentación Autobiográfica (Freud, 1925/1979) señala que se decidió por los estudios médicos después de haber considerado la posibilidad de estudiar derecho, influenciado por los intereses de un amigo, y por los suyos propios hacia la política, pero: "Entretanto, la doctrina de Darwin, reciente en aquel tiempo, me atrajo poderosamente porque prometía un extraordinario avance en la comprensión del universo, y sé que la lectura en una conferencia popular (por el profesor Carl Brühl) del hermoso ensayo de Goethe «Die Natur», que escuché poco antes de mi examen final de bachillerato, me decidió a inscribirme en medicina" (p. 8). Anzieu (1959/2004) comenta que, si bien es probable que Freud haya confundido las conferencias dominicales del profesor Brühl con una conferencias sobre Goethe, lo pertinente es observar que al leer el mencionado ensayo "sobre la naturaleza", éste usa una metáfora en la que considera a la naturaleza una madre generosa, omnisciente, todopoderosa que otorga a sus hijos favoritos el privilegio de ir a la búsqueda de sus secretos. Entonces, es posible señalar que dentro de los aspectos que influyeron en la adolescencia de Freud para optar por un cierto camino están, de manera manifiesta, el interés por comprender algunos enigmas sobre el mundo en que vivimos, entre ellos, las teorías evolucionistas de Darwin y, estrechamente relacionada con esto pero de manera latente, la curiosidad -base de las tendencias epistemofílicas y científicas- por comprender de dónde vienen los niños, de comparar la anatomía de hombres y mujeres, y la fantasía de volver a ser el hijo querido de una buena madre.

Cuando Freud emprendió sus estudios de medicina en octubre de 1873, deseaba poder realizar investigaciones biológicas de laboratorio, relacionadas con los humanos, pero no necesariamente en humanos. Casi cinco años después, afirmó, según consta en una carta para Wilhelm Knöpfmacher, con fecha del 6 de agosto de 1878 (citada por Anzieu, 1959/2004), "He cambiado de laboratorio y me preparo a ejercer mi verdadera profesión: entre desollar animales o torturar hombres, se afirma cada vez más mi preferencia por el primer término de esa alternativa" (p. 59). Efectivamente, desde 1876 y durante seis años, Freud pasó al Instituto de fisiología del profesor Ernst Wilhelm Brüke, quien le pidió que estudiase la histología de la célula nerviosa, lo cual permitió que lograra varias publicaciones sobre este tema. Dos en 1877, uno sobre el origen de las raíces nerviosas posteriores de la médula espinal del amoceto, el segundo, observaciones sobre la conformación y la estructura fina del órgano lobulado de la anguila (que ha sido considerado como testículo). Uno en 1878, sobre los ganglios espinales y la médula espinal de la lamprea. Otro en 1879, nota sobre un método de preparación anatómica del sistema nervioso. Por último, en 1882, sobre la estructura de las fibras y células nerviosas del cangrejo de río.

El periodo de 1882 a 1886 está marcado, en la vida personal de Freud, por el noviazgo y matrimonio con Martha Bernays y también fue fructífero en términos de su formación clínica como médico, pero disperso en términos científicos. En este periodo, realiza, básicamente, sus residencias médicas y algunas investigaciones neurológicas. Después de obtener su título como médico en marzo de 1881 y luego de su compromiso matrimonial en junio de 1882, Freud decide, a disgusto, abandonar el trabajo, ad honórem, que realizaba en el laboratorio. El 31 de julio de 1882, inició sus labores en el hospital de Viena. Hasta abril de 1883 estuvo en medicina general al servicio de Nothnagel (18411905). Entre mayo y octubre trabajó en el área de psiquiatría, pero ahora al servicio de Meynert (1833-1892), celebre anatomista. El último trimestre del mismo año, estuvo en dermatología. Entre enero de 1884 y febrero de 1885 en neurología, al servicio de Scholz; luego entre marzo y mayo en oftalmología, hasta agosto nuevamente en dermatología y así da el paso para vincularse con la facultad de Medicina, que le otorgará la beca para viajar a París a estudiar con Charcot.

Después de su residencia con Charcot, ya Freud no regresaría a la medicina y a la neurología como antes. Incluso, antes de este viaje, ya conocía la 
experiencia de Breuer con el caso tradicionalmente conocido como Ana O. Esa fue quizás la primera inclinación hacia lo psicológico. Pero luego del viaje a París, Freud tenía un interés especial en la psicopatología y en los factores psicógenos en la etiología de las enfermedades nerviosas, en particular, en la histeria. Esta parte de la historia biográfica de Freud es ya mucho más conocida y presentada por él mismo en su Presentación Autobiográfica (Freud, 1925/1979). Casado ya con Martha, debe buscar una mezcla entre la docencia y la práctica médica privada: la atención de pacientes. Esto favorece este periodo prepsicoanalítico en el que sigue formándose en las intervenciones terapéuticas propias de la época, de manera especial en la hipnosis, y concentrado en lograr, buscando el apoyo de Breuer, una mayor comprensión de la histeria. Toda la comprensión inicial sobre la etiología de esta enfermedad llevará a Freud a determinar las particularidades de nuestro funcionamiento psíquico a partir del planteamiento de ciertos pilares del psicoanálisis, los cuales él mismo integra en tres: "El supuesto de que existen procesos anímicos inconscientes; la admisión de la doctrina de la resistencia y la represión; la apreciación de la sexualidad y del complejo de Edipo: he ahí los principales contenidos del psicoanálisis y las bases de su teoría, y quien no pueda admitirlos todos no debería contarse entre los psicoanalistas" (Freud, 1922/1979, p. 243). Estos pilares evidencian que ya no hay una relación determinante ni con la medicina, ni con las neurociencias.

Los estudios sobre la histeria de 1895 y la comunicación preliminar de 1893 son considerados los textos que permiten el empuje final hacia el psicoanálisis. Y en 1900, La interpretación de los sueños es ya la primera obra propiamente psicoanalítica. Freud nunca abandonara completamente sus intereses previos, en particular, los neurológicos, pero éstos no serán más el centro de sus investigaciones, realizadas ahora siguiendo una metodología particular: la asociación libre.

En conclusión, el psicoanálisis se consolidó como ciencia en un momento en el cual los avances de las neurociencias no permitían dar explicación suficiente a ciertos fenómenos psíquicos. Por ello, aun cuando el camino que recorrió Freud partió de la medicina y de las investigaciones neurofisiológicas, como activamente se ha evidenciado en estas páginas, éstas no fueron suficientes para comprender fenómenos tan complejos y vigentes en su momento como el de la histeria. Se forma Freud alrededor de científicos y estudiosos como Leibniz, Charcot, Berheim, y todo esto lo conduce a buscar puentes explicativos e integradores entre lo fisiológico y lo psicológico. El concepto integrador, que descubre a través de los estudios sobre la histeria, es el de la representación y la capacidad humana de construirlas -representaciones- y desalojarlas -reprimirlas- de la propia consciencia, dejando en su lugar actos psíquicos plenos de sentido como son los síntomas. Así las cosas, Freud inicia la construcción del psicoanálisis fundamentado en elementos constitucionales humanos: los instintos, los principios que rigen el funcionamiento psíquico y nuestros potenciales psicológicos y sus vicisitudes. Este proceso se inicia prescindiendo de las explicaciones fisiológicas de estos fenómenos, las cuales Freud nunca negó, y enfatizando en las psicológicas con el ingrediente particular del inconsciente dinámico, de lo inconsciente reprimido. Además, esta construcción tiene el carácter de científica, pues se ha dado a través del empleo de una metodología particular y concreta, que consiste en el análisis de las resistencias para hacer consciente lo inconsciente reprimido, ello a través de la observación de una "regla fundamental" que, de cumplirse, lleva al paciente a la asociación libre de ideas. Esta metodología no se enriquece, ni empobrece con los hallazgos de otras disciplinas.

En particular, considero que, los principales desarrollos postfreudianos están todavía a la espera de ser investigados, están al interior del mismo psicoanálisis, y a la espera de que los psicoanalistas retomen las investigaciones propiamente psicoanalíticas, con la herramienta metodológica propia de esta ciencia, que quizás, en términos freudianos, despierta tantas "resistencias" en los analizandos, pero quizás más aún, en los psicoanalistas. Y si esto es así al interior del psicoanálisis, pues qué no decir de las resistencias que el psicoanálisis despierta en aquellos que no lo conocen profundamente. Es 
más fácil atender a los afanes postmodernos de producir conocimiento novedoso, y en muchos casos superficial, proponer nuevas disciplinas, que adentrarse en las profundidades de las ya existentes que aún tienen muchas posibilidades de desarrollos internos.

\section{Referencias}

Anzieu, D. (2004). El autoanálisis de Freud y el descubrimiento del psicoanálisis. México: Siglo XXI.

Etcheverry, J. (1979) Obras Completas de Sigmund Freud (Vol. 0). Buenos Aires: Amorrortu.

Freud, S. (1979) Sobre la dinámica de la transferencia. En J. Strachey (Ed.) y J. L. Etcheverry \& L. Wolfson (Trads.). Obras completas (Vol. 12, pp. 93. 106). Buenos Aires: Amorrortu (Trabajo original publicado en 1912).

Freud, S. (1979) Dos artículos de enciclopedia. En J. Strachey (Ed.) y J. L. Etcheverry \& L. Wolfson (Trads.). Obras completas (Vol. 18, pp. 227-254).
Buenos Aires: Amorrortu (Trabajo original publicado en 1922).

Freud, S. (1979) Presentación Autobiográfica. En J. Strachey (Ed.) y J. L. Etcheverry \& L. Wolfson (Trads.). Obras completas (Vol. 20, pp. 1-70). Buenos Aires: Amorrortu (Trabajo original publicado en 1925).

Kandel, E. (1999). Biology and the future of Psychoanalysis: A new intellectual framework for psychiatry revisited. American Journal of Psychiatry, 156 (4), 505-524.

Peled, A. (2008). NeuroAnalysis. New York: Routledge.

Solms, M. \& Turnbull, O. (2004). El cerebro y el mundo interior: una introducción a la neurociencia de la experiencia subjetiva. Bogotá: Fondo de Cultura Económica.

Solms, M. (2005). Estudios clínicos en neuropsicoanálisis. Bogotá: Fondo de Cultura Económica.

Valls, J. (2008). Diccionario Freudiano. Buenos Aires: Gaby Ediciones. 
